\title{
Insulin-Like Growth Factor 1 on the Maintenance of Ribbon Synapses in Mouse Cochlear Explant Cultures
}

\author{
Li Gao, Tomoko Kita, Tatsuya Katsuno, Norio Yamamoto, Koichi Omori \\ and Takayuki Nakagawa*
}

Department of Otolaryngology, Head and Neck Surgery, Graduate School of Medicine, Kyoto University, Kyoto, Japan

\section{OPEN ACCESS}

Edited by:

Isabel Varela-Nieto,

Consejo Superior de Investigaciones

Cientificas (CSIC), Spain

Reviewed by:

Matthew William Kelley,

National Institutes of Health $(\mathrm{NIH})$,

United States

Veronica Fuentes-Santamaría,

University of Castilla-La Mancha,

Spain

${ }^{*}$ Correspondence:

Takayuki Nakagawa

tnakagawa@ent.kuhp.kyoto-u.ac.jp

Specialty section:

This article was submitted to

Cellular Neuropathology,

a section of the journal

Frontiers in Cellular Neuroscience

Received: 10 June 2020

Accepted: 27 August 2020

Published: 08 October 2020

Citation:

Gao L, Kita T, Katsuno T,

Yamamoto N, Omori $K$ and

Nakagawa T (2020) Insulin-Like

Growth Factor 1 on the Maintenance

of Ribbon Synapses in Mouse

Cochlear Explant Cultures.

Front. Cell. Neurosci. 14:571155.

doi: 10.3389/fncel.2020.571155
Hearing loss has become one of the most common disabilities worldwide. The synaptic connections between inner hair cells (IHCs) and spiral ganglion neurons have specialized synaptic constructions, termed ribbon synapses, which are important for auditory function. The ribbon synapses in the cochlea are quite vulnerable to various insults. As such, the maintenance of ribbon synapses is important for ensuring hearing function. Insulin-like growth factor 1 (IGF1) plays a critical role in the development and maintenance of the cochlea and has the potential to protect cochlear hair cells from various insults. In this study, we examined the role of IGF1 in the maintenance of ribbon synapses in cochlear explants of postnatal day four mice. We cultured cochlear explants with an IGF1 receptor antagonist, JB1, which is an IGF1 peptide analog. Results showed that exposure to JB1 for $24 \mathrm{~h}$ resulted in the loss of ribbon synapses. After an additional 24-h culture without JB1, the number of ribbon synapses spontaneously recovered. The application of exogenous IGF1 showed two different aspects of ribbon synapses. Low doses of exogenous IGF1 promoted the recovery of ribbon synapses, while it compromised the spontaneous recovery of ribbon synapses at high doses. Altogether, these results indicate that the paracrine or autocrine release of IGF1 in the cochlea plays a crucial role in the maintenance of cochlear ribbon synapses.

Keywords: cochlea, insulin-like growth factor 1, inner hair cell, ribbon synapse, maintenance

\section{INTRODUCTION}

Hearing loss has become one of the most common disabilities worldwide. The World Health Organization (2018) estimates that hearing loss affects over 6.1\% (around 466 million people) of the world's population. The cochlea is the sensory organ responsible for auditory function. Hearing is a series of events in which sound vibrations travel into the cochlea and are then converted into electrochemical signals that are perceived by the auditory cortex. In the mammalian cochlea, the conversion is processed by mechanosensory hair cells (HCs) consisting

Abbreviations: BDNF, brain-derived neurotrophic factor; CtBP2, C-terminal-binding protein 2; HC, hair cell; IGF1, Insulin-like growth factor 1; IGF1R, Insulin-like growth factor 1 receptor; IHC, Inner hair cell; NT-3, Neurotrophin-3; OHC, Outer hair cell; P, Postnatal day; PBS, phosphate-buffered saline; rhIGF1, Recombinant human insulin-like growth factor 1; RT, Room temperature; SGN, Spiral ganglion neuron. 
of outer and inner hair cells (IHCs). Outer hair cells (OHCs) act as motor units that amplify the sound stimuli and contact type II spiral ganglion neurons (SGNs). IHCs play a key role in the process of auditory transduction. IHCs contact type I SGNs and convey electrochemical signals to the central auditory system (Hudspeth, 1997). Synaptic connections between IHCs and type I SGNs have specialized synaptic constructions, termed ribbon synapses, and are important for auditory function (Safieddine et al., 2012; Coate et al., 2019).

The ribbon synapse is an electron-dense structure associated with neurotransmitter-filled vesicles at sensory synapses in the retina, lateral line, and inner ear organs (Becker et al., 2018). Unlike conventional synapses, the ribbon synapse is specialized for the transmission of sensory information. The rapid and sustained neurotransmitter release from ribbon synapses allows for the proper transfer of sensory information to the postsynaptic receptors (Safieddine et al., 2012). The ribbon synapses in the cochlea are highly vulnerable to various insults such as ototoxic agents (Liu et al., 2013) and noise-induced injury (Kujawa and Liberman, 2009). Recent studies have demonstrated the associations between the loss of ribbon synapses and difficulties in understanding speech in noisy environments (Bharadwaj et al., 2014) and age-related hearing impairment (Viana et al., 2015). Therefore, the maintenance of ribbon synapses is crucial for ensuring normal hearing function. This encourages investigation into the protection and regeneration of cochlear ribbon synapses. Both a TrkB agonist (Meltser et al., 2014) and an AMPA receptor blocker ( $\mathrm{Hu}$ et al., 2020) have demonstrated the protection of cochlear ribbon synapses against noise trauma. In an aminoglycoside-induced degeneration model, the protection of cochlear ribbon synapses by the local application of fibroblast growth factor 22 has also been reported (Li et al., 2016). Although still controversial (Wagner and Shin, 2019), the capacity to regenerate ribbon synapses in the mammalian cochlea was demonstrated in vitro (Wang and Green, 2011; Yamahara et al., 2019) and in vivo (Wan et al., 2014; Suzuki et al., 2016).

Insulin-like growth factor 1 (IGF1), once known as somatomedin $\mathrm{C}$, is a hormone similar in molecular structure to insulin. IGF1 is essential for controlling cell proliferation, differentiation, and apoptosis in various tissues and organs, including the inner ears (Varela-Nieto et al., 2007; Okano et al., 2011; Allahdadi et al., 2019). IGF1 has the potential to protect HCs from excessive noise (Iwai et al., 2006; Lee et al., 2007), aminoglycoside antibiotics (Hayashi et al., 2017), ischemic trauma (Fujiwara et al., 2008), and surgical invasion (Yamahara et al., 2018). Also, the therapeutic effects of recombinant human IGF1 (rhIGF1) on sudden sensorineural hearing loss refractory to systemic corticosteroids have been confirmed in clinical studies (Nakagawa et al., 2010, 2014, 2016). IGF1 performs its functions by binding to its tyrosine kinase receptor (IGF1R). In the cochlea, IGF1R is expressed on the surface of OHCs, IHCs, and all supporting cells, including pillar cells, Hensen's and Claudius' cells, inner sulcus cells, and Deiters' cells (Okano et al., 2011; Hayashi et al., 2013), as well as SGNs (Sanchez-Calderon et al., 2010). Our previous study confirmed that IGF1 induced the regeneration of ribbon synapses in cochlear explant cultures of postnatal day (P) two mice (Yamahara et al., 2019). In this study, we examined the roles of IGF1 signaling in the maintenance of cochlear ribbon synapses using explant cultures. For the pharmacological inhibition of IGF1Rs, we used a competitive IGF1R antagonist, JB1, which is an IGF1 peptide analog. JB1 is a 12-amino acid cyclic peptide that inhibits the binding of IGF1 to its receptor (Pietrzkowski et al., 1992). We further confirmed the potential for spontaneous recovery of ribbon synapses and the additional effect of exogenous IGF1 in cochlear explants that had previously been damaged by JB1.

\section{MATERIALS AND METHODS}

\section{Animals}

Institute of Cancer Research (ICR) mice aged postnatal day 4 were purchased from Japan SLC (Hamamatsu, Japan). All animal procedures were performed following the NIH Guide for the Care and Use of Laboratory Animals (NIH Publications No. 8023, revised 1978) and were approved by the Animal Research Committee of Kyoto University Graduate School of Medicine (Med Kyo 17,114, 18,117, 19,536).

\section{Explant Cultures}

Cochlear explant cultures were performed as previously described (Yamahara et al., 2019). Briefly, P4 mice were killed with carbon dioxide $\left(\mathrm{CO}_{2}\right)$, after which they were decapitated. The cochleae were removed from the temporal bones and dissected in ice-cold sterile $0.1 \mathrm{M}$ phosphate-buffered saline (PBS). The surrounding bony capsules were removed, and cochlear sensory epithelia were carefully dissected to preserve afferent connections with SGNs. To minimize any differences related to the region of cochlea under study, we only used the middle portion (40-60\% from the apex) of the cochlear sensory epithelia. The samples were placed on cell culture inserts (Becton Dickinson, Franklin Lake, NJ, USA) and incubated in Dulbecco's modified Eagle's medium (DMEM; Sigma-Aldrich Inc., St. Louis, MO, USA) supplemented with 6 g/l D-glucose (Wako Pure Chemicals, Osaka, Japan) and $0.15 \mathrm{~g} / \mathrm{l}$ penicillin $\mathrm{G}$ (Wako Pure Chemicals, Osaka, Japan) at $37^{\circ} \mathrm{C}$ in a humidified atmosphere with $5 \% \mathrm{CO}_{2}$. To prevent any effects caused by serum growth factors or hormones, serum-free medium was used in all experiments. Overnight pre-cultures were established to stabilize the cochlear explants.

\section{Pharmacological Inhibition by JB1}

After the overnight pre-cultures, cochlear explants were exposed to JB1 (Cat. No. J3705; Sigma-Aldrich Inc., St. Louis, MO, USA) at a concentration of $25,50,100$, or $150 \mu \mathrm{g} / \mathrm{ml}$ for $24 \mathrm{~h}(n=5$ for each). Cochlear explants that were cultured without exposure to JB1 were used as controls. Cultured samples were histologically assessed for the number of IHCs, density of SGNs, as well as number of density of SGNs, as well as number of presynaptic ribbons, and postsynaptic receptor patches per IHC.

\section{Spontaneous Recovery of Ribbon Synapses}

To assess the capacity for spontaneous recovery of ribbon synapses, we used cochlear explants that were pre-treated with 
$50 \mu \mathrm{g} / \mathrm{ml} \mathrm{JB} 1$ for $24 \mathrm{~h}$. After rinsing for $5 \mathrm{~min}$ thrice with culture media, cochlear explants $(n=9)$ were incubated in the culture media for another $24 \mathrm{~h}$ without being exposed to JB1. The cochlear explants exposed to JB1 for $24 \mathrm{~h}$ were used as controls $(n=9)$. The number of presynaptic ribbons and postsynaptic receptor patches per IHC was compared between the two groups.

\section{Effects of Exogenous IGF1 on Ribbon Synapses}

To determine the effect of exogenous IGF1 on the recovery of presynaptic ribbons and postsynaptic receptor patches, cochlear explants that had been exposed to $50 \mu \mathrm{g} / \mathrm{ml} \mathrm{JB1}$ for $24 \mathrm{~h}$ were incubated with culture media supplemented with rhIGF1 (Orphan Pacific Pharma, Tokyo, Japan) at a concentration of 0 , $0.1,0.15,0.2,0.5,1.0$, or $5.0 \mu \mathrm{g} / \mathrm{ml}(n=5-10)$ for $24 \mathrm{~h}$. The specimens were then histologically examined.

\section{Immunohistochemistry}

Specimens were fixed in $4 \%$ paraformaldehyde in phosphate buffer (0.1 M; pH 7.4) for $15 \mathrm{~min}$ at room temperature (RT) and then rinsed with PBS. We analyzed ribbon synapses by immunohistochemical staining for C-terminal-binding protein 2 (CtBP2) and ionotropic glutamate receptor-2 (GluA2), as previously described (Liberman and Liberman, 2016; Suzuki et al., 2016). After blocking with $10 \%$ goat serum in $0.2 \%$ Triton $^{\mathrm{TM}} \mathrm{X}-100$ for $30 \mathrm{~min}$ at RT, we incubated the specimens at $37^{\circ} \mathrm{C}$ overnight with the primary antibodies shown in Table 1 diluted in $10 \%$ goat serum in PBS. We incubated samples with primary antibody species-specific secondary antibodies with Alexa Fluor ${ }^{\circledR}$ conjugates (Table 1) for $1 \mathrm{~h}$ at RT.

\section{Presynaptic Ribbon and Postsynaptic Receptor Patch Counting}

Cochlear specimens were imaged with a Leica TCS-SPE confocal microscope (Leica Microsystems, Wetzlar, Germany) equipped with 405, 488, 532, and $635 \mathrm{~nm}$ solid-state lasers. Scanning was sequential with a $2.5 \mu \mathrm{s}$ dwell time. Images were sampled at a resolution of 512 by 512 pixels with a $63 \times$ oil immersion (NA 1.3) objective, a $1.5 \times$ software zoom, and a $z$-step size of $0.17 \mu \mathrm{m}$. A series of images contained at least 10 IHCs from the cuticular plate to the base. For SGN density analysis, a $z$-step size of $0.5 \mu \mathrm{m}$ was applied to include the soma and SGN afferent dendrites. Imaris 7.4.2 software (BitPlane AG, Zurich, Switzerland) was used to perform three-dimensional reconstructions and spot analyses to count the number of presynaptic ribbons and postsynaptic receptor, as previously described (Fogarty et al., 2013). Puncta in each IHC with immunoreactivity for $\mathrm{CtBP} 2$ were counted as presynaptic ribbons. Puncta in each IHC with immunoreactivity for both $\mathrm{CtBP} 2$ and GluA2 were defined as postsynaptic receptor patches. For the spot analysis, the minimum diameter of a punctum was defined as $0.7 \mu \mathrm{m}$. For each sample, the number of presynaptic ribbons and postsynaptic receptor patches was manually counted in 10 IHCs.

\section{Statistical Analysis}

Student's $t$-test or one-way analysis of variance (ANOVA) with Tukey post hoc test was used. Statistical analysis was performed using GraphPad Prism v6.0 for Mac (GraphPad, San Diego, CA, USA) and SPSS 25 version for Mac (SPSS Inc., Chicago, IL, USA). The data are expressed as the mean \pm standard deviation. Differences with $p$-values $<0.05$ were considered statistically significant.

\section{RESULTS}

\section{An IGF1R Antagonist Induced Loss of Ribbon Synapses in Cochlear Explant Cultures}

To assess the effect of pharmacological inhibition of IGF1R on the maintenance of cochlear ribbon synapses, we exposed cochlear explant cultures of P4 mice to an IGF1R antagonist, JB1, at different concentrations, ranging from 0 to $150 \mu \mathrm{g} / \mathrm{ml}$, for $24 \mathrm{~h}$ (Figure 1A). All assays were performed using the middle portion (40-60\% from the apex) of the cochlea because the numbers of ribbon synapses per IHC varied according to the location in the cochlear turns (Meyer et al., 2009). Immunohistochemistry for CtBP2 for labeling presynaptic ribbons and GluA2 for labeling postsynaptic receptor patches was used to assess the ribbon synapses histologically. The location of IHCs was determined by immunostaining for myosin VIIa. Immunohistochemical analyses for CtBP2 and GluA2 demonstrated ribbon synapses in control specimens that were cultured without JB1 (Figures 1B-D). A decrease in the number of ribbon synapses was observed in specimens cultured with JB1 at concentrations of 50 (Figures 1E-G) and 150 $\mu \mathrm{g} / \mathrm{ml}$ (Figures $\mathbf{1 H}-\mathbf{J}$ ). We quantified the number of CtBP2positive puncta (as presynaptic ribbons) and CtBP2 and GluA2 co-stained puncta (as postsynaptic receptor patches) per IHC, respectively. JB1 had significant effects on both presynaptic ribbons ( $p<0.001$ by one-way ANOVA) and postsynaptic

TABLE 1 | The information of antibodies.

\begin{tabular}{lcll}
\hline Antibody & Working dilution & Cat. No. & Company \\
\hline Rabbit polyclonal anti-myosin 7a & $1: 500$ & $25-6790$ & Proteus BioScience, Ramona, CA, USA \\
Mouse monoclonal immunoglobulin G (lgG)1 anti-CtBP2 & $1: 250$ & 612044 & BD Biosciences, Franklin Lakes, NJ, USA \\
Mouse monoclonal lgG2a anti-GluA2 & $1: 2,000$ & MAB397 & Merck Millipore, Burlington, MA, USA \\
Chicken polyclonal anti-neurofilament-H & $1: 1,000$ & AB5539 & Merck Millipore, Burlington, MA, USA \\
Alexa Fluor 405 Goat anti-rabbit lgG $(\mathrm{H}+\mathrm{L})$ & $1: 1,000$ & $\mathrm{~A}-31556$ & Thermo Fisher Scientific, Waltham, MA, USA \\
Alexa Fluor 568 Goat anti-mouse lgG1 & $1: 1,000$ & $\mathrm{~A}-21124$ & Thermo Fisher Scientific, Waltham, MA, USA \\
Alexa Fluor 488 Goat anti-mouse lgG2a & $1: 1,000$ & $\mathrm{~A}-21131$ & Thermo Fisher Scientific, Waltham, MA, USA \\
Alexa Fluor 647 Goat anti-chicken lgG $(\mathrm{H}+\mathrm{L})$ & $1: 1,000$ & $\mathrm{~A}-21449$ & Thermo Fisher Scientific, Waltham, MA, USA
\end{tabular}




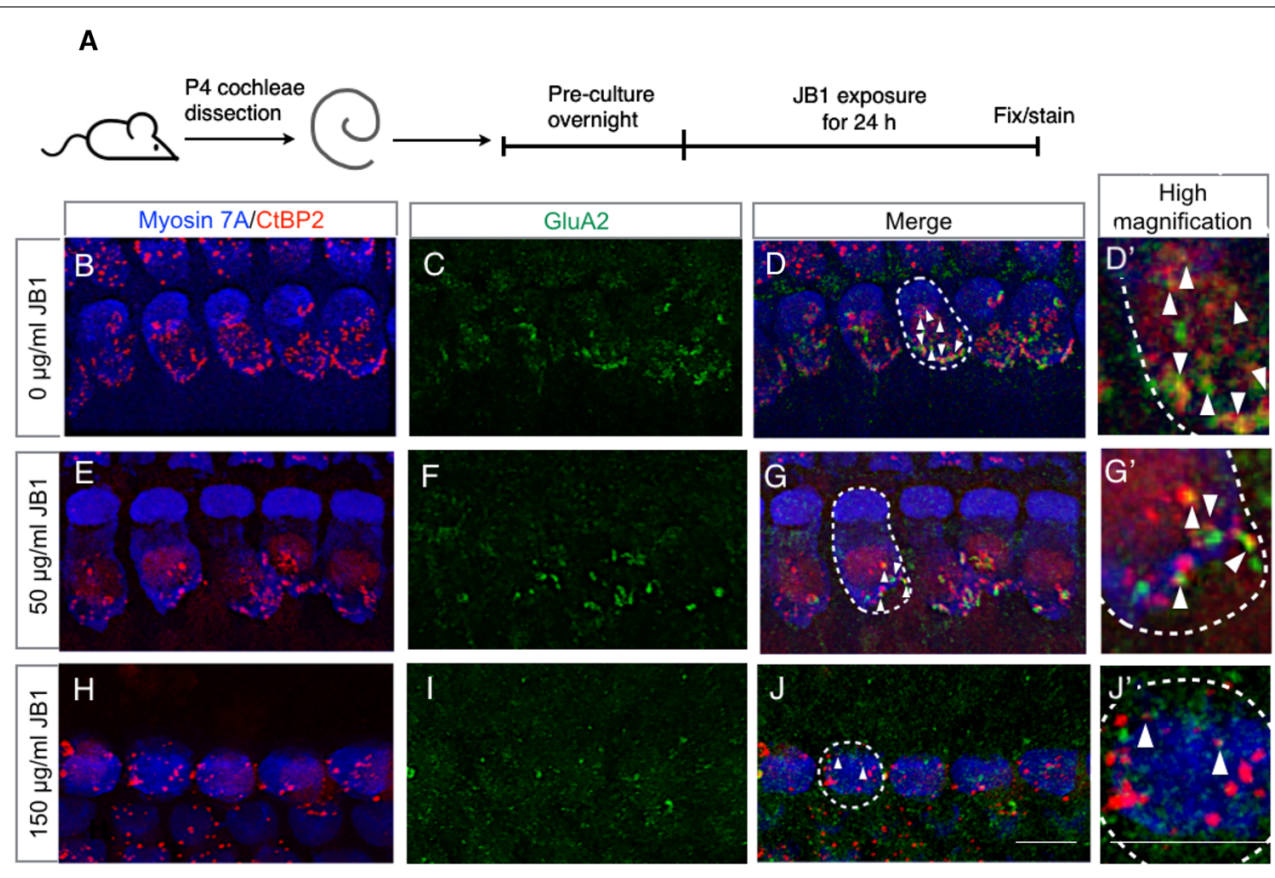

K Numbers of presynaptic ribbon

L Numbers of postsynaptic receptor patch
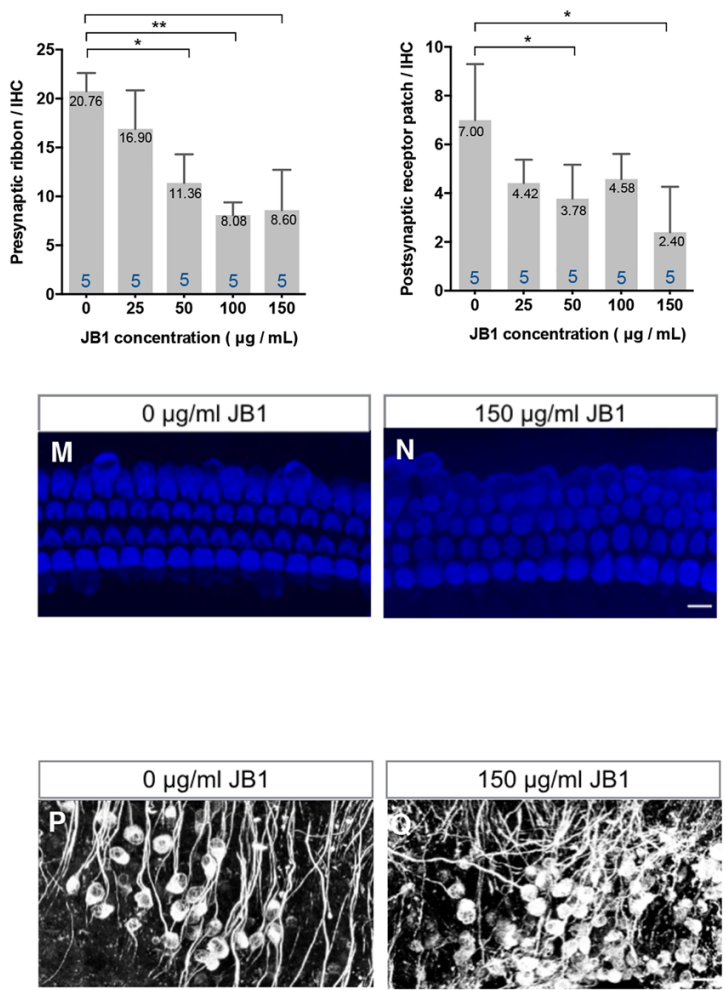

O Numbers of inner hair cells after 24-h JB1 exposure

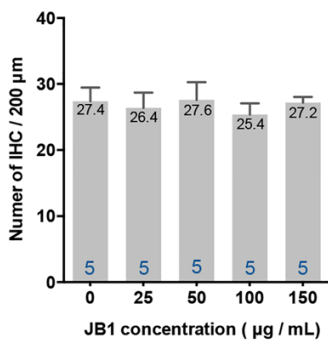

R Density of spiral ganglion neurons

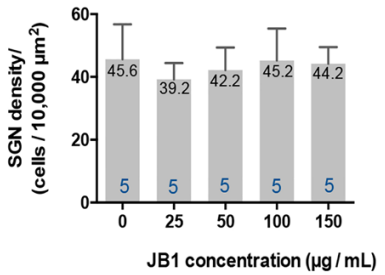

FIGURE 1 | An insulin-like growth factor 1 receptor (IGF1R) antagonist JB1 induced the loss of ribbon synapses in cochlear explant cultures. Cochlear explants of P4 mice were exposed to JB1 at a concentration of 25, 50, 100, or $150 \mu \mathrm{g} / \mathrm{ml}$ for $24 \mathrm{~h}$ (A). (B-D) Maximal-intensity projection images with z-stack of ribbon synapses in control specimens that were cultured without JB1. Panels (E-J) are maximal-intensity projection images of the degeneration of ribbon synapses in specimens cultured with JB1 at concentrations of 50 and $150 \mu \mathrm{g} / \mathrm{ml}$, respectively. Arrows show the postsynaptic receptor patches and dotted lines indicate the location of an IHC. (K) JB1 showed significant effects 
FIGURE 1 | Continued

on both presynaptic ribbons ( $p<0.001$ by one-way ANOVA) and postsynaptic receptor patches (L; $p=0.004$ by one-way ANOVA). Tukey's post hoc test revealed significant losses at concentrations of $50(p=0.001)$, $100(p<0.001)$, and $150 \mu \mathrm{g} / \mathrm{ml}(p<0.001)$ in the number of presynaptic ribbons, and at concentrations of $50(p=0.032)$ and $150 \mu \mathrm{g} / \mathrm{ml}(p=0.002)$ in the number of postsynaptic receptor patches, in comparison with controls which were cultured without JB1. (M-R) No inner hair cell $(\mathrm{IHC})$ or spiral ganglion neuron (SGN) loss was found at any JB1 concentrations $(p=0.428$ by one-way ANOVA for $\mathrm{IHC}, p=0.730$ by one-way ANOVA for SGN). Scale bars: $10 \mu \mathrm{m}$. Data are expressed as mean (digits at the top of each bar) \pm SD. The digits at the bottom of each bar represent the sample number. ${ }^{*} p<0.05 ;{ }^{* *} p<0.01$ with Tukey's post hoc test. P4, postnatal day 4; IHC, inner hair cell; SGN, spiral ganglion neuron; SD, standard deviation; ANOVA, analysis of variance.

receptor patches ( $p=0.004$ by one-way ANOVA), respectively (Figures 1K,L). Tukey's post hoc test revealed significant losses at concentrations of $50(p=0.001), 100(p<0.001)$ and 150 $\mu \mathrm{g} / \mathrm{ml}(p<0.001)$ in the number of presynaptic ribbons, and at concentrations of $50(p=0.032)$ and $150 \mu \mathrm{g} / \mathrm{ml}(p=0.002)$ in the number of postsynaptic receptor patches, in comparison with controls which were cultured without JB1 (Figures 1K,L). No significant loss of IHC (Figures 1M-R) or SGN (Figures 1P-R) was found at any JB1 concentration ( $p=0.428$ by one-way ANOVA for IHC, $p=0.730$ by one-way ANOVA for SGN). These findings demonstrate that the pharmacological inhibition of IGF1R caused a decrease in both presynaptic ribbons and postsynaptic receptor patches, suggesting that IGF1 is necessary for the maintenance of ribbon synapses in cochlear explant cultures.

\section{Spontaneous Recovery of Ribbon Synapses}

To test the spontaneous recovery capacity of ribbon synapses, we performed an additional 24-h culture without the presence of JB1 following a 24-h exposure period to JB1 at a concentration of $50 \mu \mathrm{g} / \mathrm{ml}$ (Figure 2A). Compared with cochlear explants exposed to JB1 for $24 \mathrm{~h}$ (Figures 2B-D), cochlear explants incubated for an additional $24 \mathrm{~h}$ without being exposed to JB1 tended to recover their ribbon synapses (Figures 2E-G). Statistical analyses using unpaired $t$-test demonstrated a significant increase in the numbers of both presynaptic ribbons (Figure 2H, $p<0.001$, student's $t$-test) and postsynaptic receptor patches (Figure 2I, $p=0.046$, student's $t$-test) in specimens following an additional 24-h culture. These findings indicate that cochlear explants of P4 mice have the capacity for spontaneous recovery of both presynaptic ribbons and postsynaptic receptor patches following their loss because of the pharmacological inhibition of IGF1R.

\section{Effects of Exogenous IGF1 on the Recovery of Cochlear Ribbon Synapses}

To assess the additional effects of exogenous IGF1 on the recovery of ribbon synapses, cochlear explants that had been damaged by $24-\mathrm{h}$ exposure to $50 \mu \mathrm{g} / \mathrm{ml} \mathrm{JB1}$ were incubated with culture media supplemented with rhIGF1 for $24 \mathrm{~h}$ (Figure 3A). Compared with the specimens following an additional 24-h culture without supplementation of rhIGF1 (Figures 3B-D), further increase in the number of ribbon synapses was observed in the specimens following an additional 24-h culture with 0.2 $\mu \mathrm{g} / \mathrm{ml}$ rhIGF1 (Figures 3E-G). However, in those following an additional 24-h culture with $5 \mu \mathrm{g} / \mathrm{ml}$ rhIGF1 (Figures $3 \mathbf{H}-\mathbf{J}$ ), a decrease in the number of ribbon synapses was observed. Quantitative analyses showed significant effects of exogenous rhIGF1 on the numbers of both presynaptic ribbons $(p<0.001$, one-way ANOVA, Figure $3 \mathbf{K}$ ) and postsynaptic receptor patches $(p<0.001$, one-way ANOVA, Figure 3L). Significant differences were identified in the number of presynaptic ribbons between 0 and 0.2 ( $p=0.034$, Tukey post hoc test), 1 ( $p=0.017$, Tukey post hoc test) or $5 \mu \mathrm{g} / \mathrm{ml}$ ( $p=0.010$, Tukey post hoc test), and in the number of postsynaptic receptor patches between 0 and 0.2 ( $p=0.020$, Tukey post hoc test) or $5 \mu \mathrm{g} / \mathrm{ml}(p=0.012$, Tukey post hoc test). In summary, exogenous IGF1 at concentrations $<0.5$ $\mu \mathrm{g} / \mathrm{ml}$ exerted positive effects on the recovery of ribbon synapses, but high concentrations diminished spontaneous recovery. We tested the effect of rhIGF1 at a concentration of $5 \mu \mathrm{g} / \mathrm{ml}$ on the survival of IHCs or SGNs. The results demonstrated no significant IHC or SGN loss in specimens treated with 5 $\mu \mathrm{g} / \mathrm{ml}$ rhIGF1 compared with those cultured without rhIGF1 (Supplementary Figure S1). These findings indicate that the precise regulation of IGF1 levels may be required for the maintenance of ribbon synapses in cochlear explant cultures.

\section{DISCUSSION}

In this study, we used the IGF1R antagonist JB1 to pharmacologically inhibit IGF1 signaling. JB1 is a highly selective IGF-IR antagonist, which interferes with the efficacy of IGF1 by competitively inhibiting the binding of IGF1 to IGF1R (Pietrzkowski et al., 1992). Our previous study demonstrated the efficacy of JB1 in attenuating IGF1-mediated regeneration of ribbon synapses in P2 cochlear explants that had been damaged by excitatory amino acids (Yamahara et al., 2019). JB1 has also been used to antagonize IGF1 effects in various organs including the hippocampus (Nelson et al., 2014) and retina (Landi et al., 2009). The present results demonstrated that JB1 decreased the number of both presynaptic ribbons and postsynaptic receptor patches in a dose-dependent manner, indicating that IGF1 is an inevitable factor for the maintenance of ribbon synapses in cochlear explants. Our previous study (Yamahara et al., 2019) used explant cultures of P2 mouse cochleae, while in the present study, we used P4 mouse cochleae for a couple of reasons. One is the maturation process of ribbon synapses. The period from $\mathrm{P} 0$ to $\mathrm{P} 12$ is an active phase for the maturation of ribbon synapses (Michanski et al., 2019), indicating that ribbon synapses in P4 mice are more mature than those in P2 mice. On the other hand, there is a technical limitation in the preparation of cochlear explant culture. It is difficult to dissect out cochlear sensory epithelia from P5 or older mouse cochleae. Based on these issues, P4 mice were chosen for this study. Ribbon synapses in P4 mouse cochleae are still immature, which can be a limitation of the present study.

Previous studies have revealed the importance of IGF1 for the maturation of cochleae after birth (Camarero et al., 2001, 2002). In homozygous mutants, $\mathrm{IGF} 1^{-l-}$ mice, no significant 


\section{A}
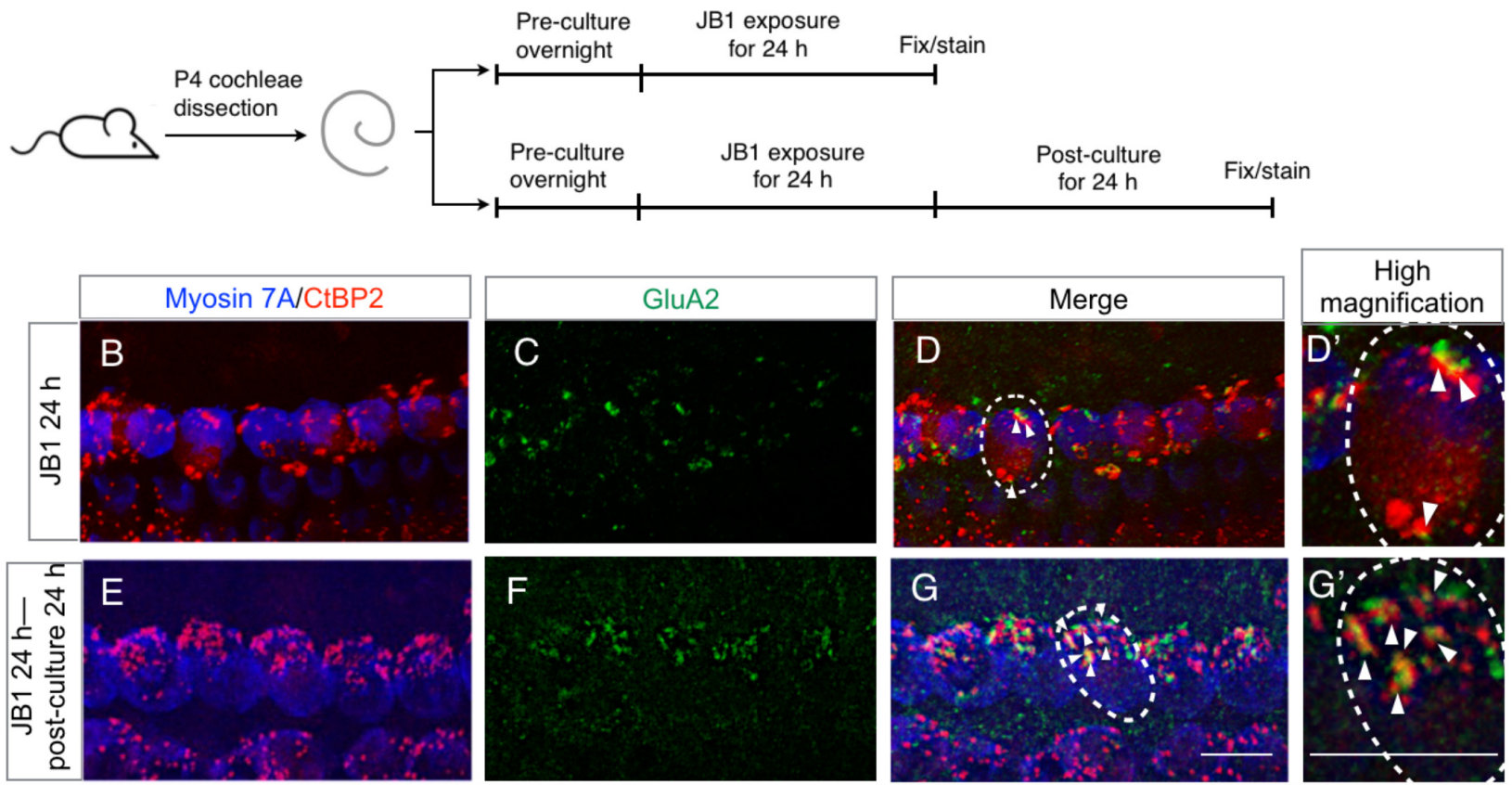

H Numbers of presynaptic ribbon

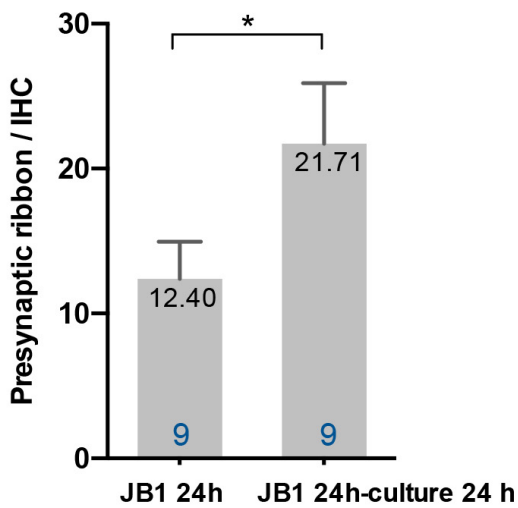

I Numbers of postsynaptic receptor patch

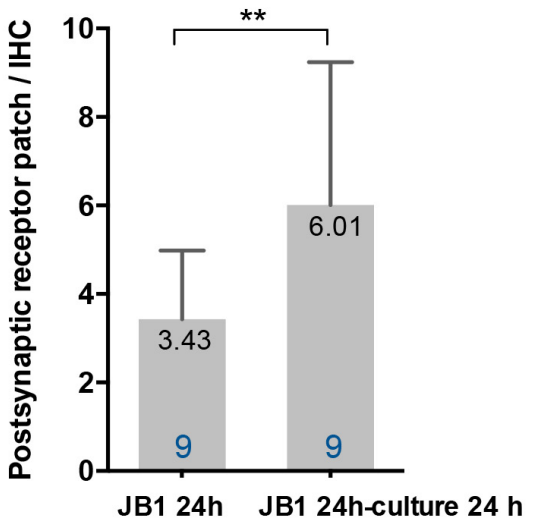

FIGURE 2 | Spontaneous recovery of ribbon synapses. Cochlear explants from P4 mice were exposed to $50 \mu \mathrm{g} / \mathrm{ml} \mathrm{JB1}$ for $24 \mathrm{~h}$, with or without an additional $24 \mathrm{~h}$ of culture (A). (B-D) Maximal-intensity projection images with $z$-stack of the immunostaining results of specimens immediately after 24-h JB1 exposure. (E-G) Maximal-intensity projection images of the specimens following an additional 24-h culture. Arrows show the postsynaptic receptor patches and dotted lines indicate the location of an IHC. $\mathbf{( H )}$ The number of presynaptic ribbons $(p<0.001$ by unpaired $t$-test) and postsynaptic receptor patches $(\mathbf{I} ; p=0.046$ by unpaired $t$-test) in the specimens were significantly increased after an additional 24-h culture. Scale bars: $10 \mu \mathrm{m}$. Data are expressed as mean (digits at the top of each bar) \pm SD. The digits at the bottom of each bar represent the sample number. ${ }^{\star} p<0.01 ;{ }^{* \star} p<0.05$ by an unpaired $t$-test. P4, postnatal day 4 ; IHC, inner hair cell; SD,

standard deviation.

loss of SGNs was identified at P5, while SGN numbers and sizes were statistically significantly decreased at P20. Moreover, cochlear volumes were also revealed to have the same trend (Camarero et al., 2001). Abnormal innervation of SGN afferent dendrites was observed at P20, but not at P5 (Camarero et al., 2002). Also, IGF1-deficient mice presented profound deafness from 1 month of age onwards, without any obvious worsening of hearing parameters with aging (Riquelme et al., 2010). These findings suggest the importance of IGF1 for the maintenance and maturation of the synaptic contacts between IHCs and SGNs.

IGF1 is mainly produced in the liver and exerts its function through endocrine, autocrine, and paracrine mechanisms (Jenkins and Bustin, 2004). IGF1 is also synthesized in the developing and neonatal cochleae 

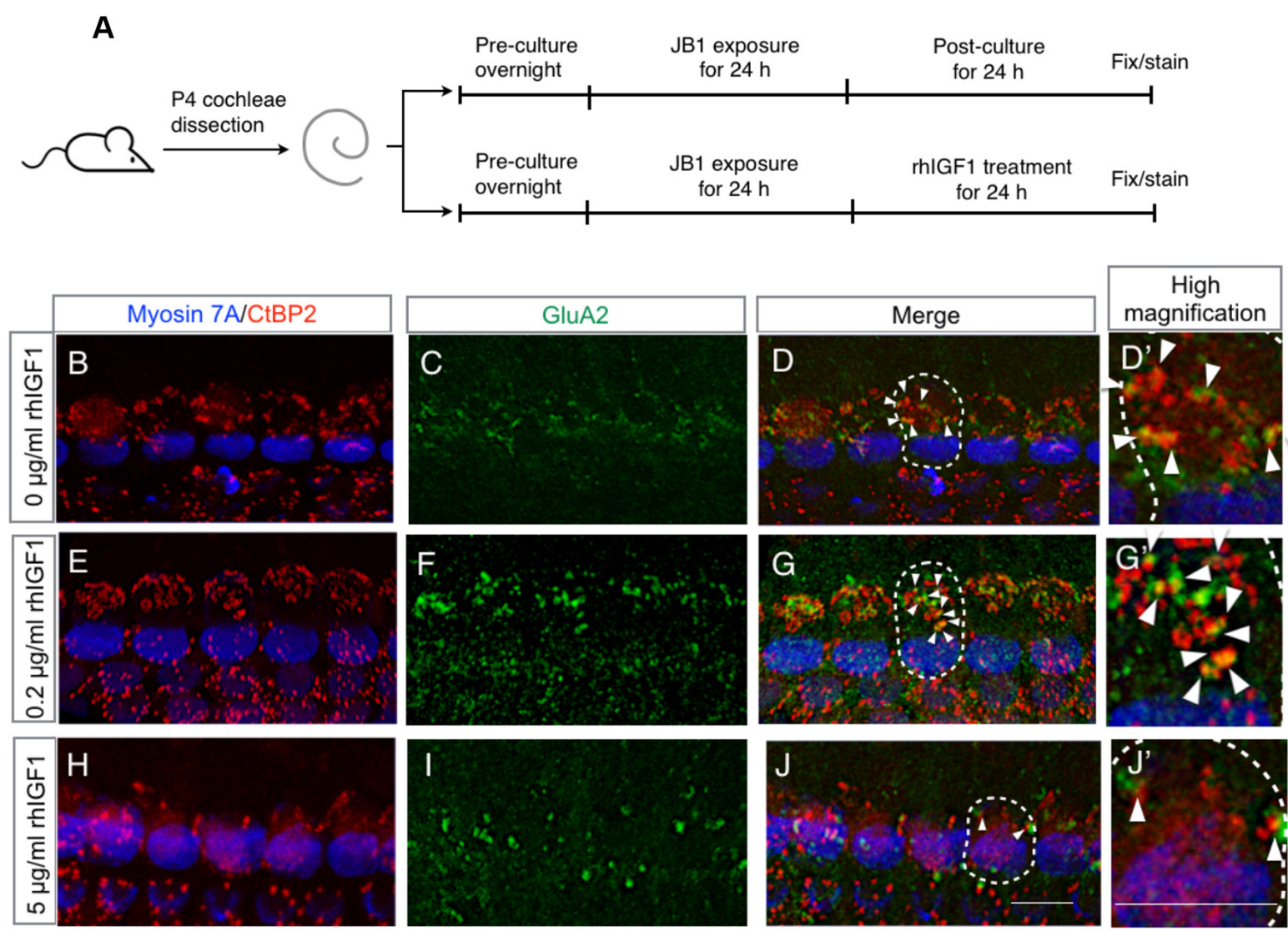

K Numbers of presynaptic ribbon

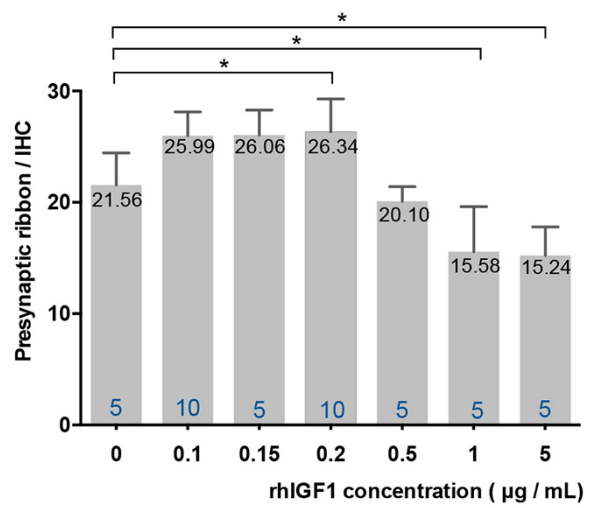

L Numbers of postsynaptic receptor patch

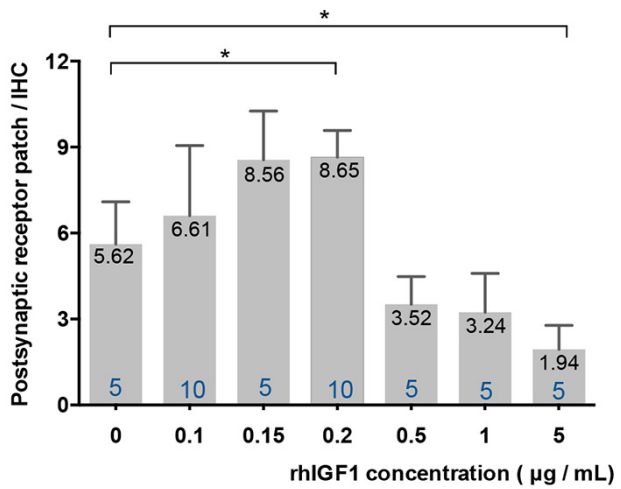

FIGURE 3 | Effect of exogenous IGF1 on the recovery of ribbon synapses. Cochlear explants from P4 mice were exposed to $50 \mu \mathrm{g} / \mathrm{ml} \mathrm{JB1}$ for $24 \mathrm{~h}$, after which they were incubated with culture media supplemented with rhlGF1 at a concentration of 0.1, 0.15, 0.2, 0.5, or 1, $5 \mu \mathrm{g} / \mathrm{ml}$ for $24 \mathrm{~h}$ (A). (B-D) Maximal-intensity projection images with $z$-stack of the immunostaining images of specimens cultured without rhlGF1. Panels (E-J) are maximal-intensity projection images of the recovery of ribbon synapses in specimens cultured with $50 \mu \mathrm{g} / \mathrm{ml} \mathrm{JB} 1$ for $24 \mathrm{~h}$, followed by rhlGF1 treatment for $24 \mathrm{~h}$ at concentrations of 0.2 and $5 \mu \mathrm{g} / \mathrm{ml}$, respectively. Arrows show the postsynaptic receptor patches and dotted lines indicate the location of an IHC. Exogenous rhIGF1 showed significant effects on the number of both presynaptic ribbons (K; $p<0.001$ by one-way ANOVA) and postsynaptic receptor patches (L; $p<0.001$ by one-way ANOVA). Tukey's post hoc test revealed significant difference at concentrations of $0.2(p=0.034), 1(p=0.017)$, and $5 \mu \mathrm{g} / \mathrm{ml}(p=0.010)$ in the number of presynaptic ribbons, and at concentrations of $0.2(p=0.020)$ and $5 \mu \mathrm{g} / \mathrm{ml}(p=0.012)$ in the number of postsynaptic receptor patches, in comparison with controls which were cultured in 0 $\mu \mathrm{g} / \mathrm{ml}$ rhlGF1. Scale bars: $10 \mu \mathrm{m}$. Data are expressed as mean (digits at the top of each bar) \pm SD. The digits at the bottom of each bar represent the sample number. ${ }^{*} p<0.05$ by one-way ANOVA, followed by Tukey's post hoc test. P4, postnatal day 4; IHC, inner hair cell; SD, standard deviation; rhlGF1, recombinant human insulin-like growth factor-1; ANOVA, analysis of variance. 
(Sanchez-Calderon et al., 2010; Okano et al., 2011), indicating the existence of paracrine or autocrine systems for IGF1 in the cochlea. In this study, we observed the spontaneous recovery of both presynaptic ribbons and postsynaptic patches after their loss because of the pharmacological inhibition of IGF1R. Therefore, we presume that the paracrine or autocrine systems in the cochlea may contribute to the spontaneous recovery of ribbon synapses in cochlear explants that had been damaged by JB1.

Neurotrophin-3 (NT-3) is a potent factor for the maintenance and regeneration of cochlear ribbon synapses (Wang and Green, 2011; Wan et al., 2014). The selective blockade of endogenous NT-3 signaling reduced the regeneration of axons and postsynaptic densities in vitro (Wang and Green, 2011). Endogenous NT-3 promoted the recovery of cochlear ribbon synapses after acoustic trauma in vivo (Wan et al., 2014). Brainderived neurotrophic factor (BDNF) also plays a role in the development and maintenance of ribbon synapses (Zuccotti et al., 2012; Meltser et al., 2014; Wan et al., 2014). In this study, the selective blockade of IGF1R induced the significant loss of cochlear ribbon synapses, suggesting that endogenous NT-3 or BDNF in $\mathrm{P} 4$ cochlear explants could not compensate for the loss of IGF1 signaling. In contrast, other factors, including NT-3 and BDNF, may be involved in the process of spontaneous recovery of ribbon synapses that were observed in this study.

In our previous studies using cochlear explants of neonatal mice, exogenous rhIGF1 had protective effects against aminoglycoside ototoxicity on IHCs at concentrations ranging from 0.1 to $1 \mu \mathrm{g} / \mathrm{ml}$, and on $\mathrm{OHCs}$ at concentrations ranging from $10^{-4}$ to $1 \mu \mathrm{g} / \mathrm{ml}$ (Hayashi et al., 2013; Yamahara et al., 2017). For the regeneration of ribbon synapses after excitotoxic insults, $1 \mu \mathrm{g} / \mathrm{ml}$ exogenous rhIGF1 showed significant effects, but $5 \mu \mathrm{g} / \mathrm{ml}$ did not (Yamahara et al., 2019). Based on these findings, we used exogenous rhIGF1 at concentrations ranging from 0.1 to $5 \mu \mathrm{g} / \mathrm{ml}$ in this study. These results demonstrated the positive effects on the recovery of both presynaptic ribbons and postsynaptic receptor patches at concentrations of rhIGF1 ranging from 0.1 to $0.2 \mu \mathrm{g} / \mathrm{ml}$, and negative effects at a concentration of 1 and $5 \mu \mathrm{g} / \mathrm{ml}$. Compared with our previous study using cochlear explants that were damaged by excitatory amino acids (Yamahara et al., 2019), the concentration of rhIGF1 required to promote recovery is lower, which could be due to differences in the method used to induce damage. In contrast to the present results, after the exposure to excitatory amino acids, no spontaneous recovery of ribbon synapses was observed, indicating the lack of endogenous IGF1 production. In the present study, exogenous rhIGF1 exhibited bell-shaped dose-response curves in the numbers of presynaptic ribbons and postsynaptic receptor patches. This type of dose-response curve is common with endocrine disruption (Vandenberg et al., 2012). Some hormones, including androgen and estradiol, show agonist effects at low concentrations and antagonist effects at high concentrations. There are several mechanisms for a bell-shaped dose-response curve, including actions at multiple targets, cytotoxicity, receptor selectivity, receptor down-regulation and desensitization, receptor competition, and endocrine negative feedback loops (Vandenberg et al., 2012). IGF1 is similar in molecular structure to insulin and has a low affinity to insulin receptors. At high concentrations, IGF1 activates insulin receptor-mediated signaling. Altogether, the precise regulation of IGF1 levels may be necessary for the maintenance of cochlear ribbon synapses.

In conclusion, in the current study, we demonstrated that the pharmacological inhibition of IGF1R reduced the number of ribbon synapses in cochlear explant cultures of $\mathrm{P} 4$ mice, pointing to the importance of IGF1 for the maintenance of ribbon synapses. Surprisingly, the number of ribbon synapses recovered spontaneously following an additional $24 \mathrm{~h}$ of culture, pointing to the contribution of endogenous IGF1 to the maintenance of cochlear ribbon synapses. This confirmation of the effects of exogenous rhIGF1 points to the precise regulation of IGF1 levels for the maintenance of cochlear ribbon synapses. However, ribbon synapses in $\mathrm{P} 4$ cochleae are still in their maturation process (Michanski et al., 2019), which is a limitation of this study. We will investigate the role of IGF1 in the maintenance of cochlear ribbon synapses in mature animals in vivo shortly.

\section{DATA AVAILABILITY STATEMENT}

The raw data supporting the conclusions of this article will be made available by the authors, without undue reservation.

\section{ETHICS STATEMENT}

The animal study was reviewed and approved by the Animal Research Committee of Kyoto University Graduate School of Medicine.

\section{AUTHOR CONTRIBUTIONS}

TN designed the study. LG, TKi and TKa performed the experiments. LG and TN analyzed the data and wrote the manuscript. NY and KO performed a critical review.

\section{FUNDING}

This work was supported by KAKENHI (17k11325 and $16 \mathrm{H} 06280$ ), from the Japan Society for the Promotion of Science, AMED (Japan Agency for Medical Research and Development) under Grant No. 17ak0101042h0003, and Grant-in-Aid for Scientific Research on Innovative AreasPlatforms for Advanced Technologies and Research Resources "Advanced Bioimaging Support".

\section{ACKNOWLEDGMENTS}

We would like to thank Kohei Yamahara (Kyoto University) for valuable suggestions on the design of experiments, Jun Suzuki (Tohoku University) for technical advice for immunostaining, and Editage (www.editage.com) for English language editing. 


\section{SUPPLEMENTARY MATERIAL}

The Supplementary Material for this article can be found online at: https://www.frontiersin.org/articles/10.3389/fncel.2020.5711 55/full\#supplementary-material.

FIGURE S1 | Effects of exogenous IGF1 on the survival of inner hair cells (IHCS) and spiral ganglion neurons (SGNs). Cochlear explants from P4 mice were

\section{REFERENCES}

Allahdadi, K. J., de Santana, T. A., Santos, G. C., Azevedo, C. M., Mota, R. A., Nonaka, C. K., et al. (2019). IGF-1 overexpression improves mesenchymal stem cell survival and promotes neurological recovery after spinal cord injury. Stem Cell Res. Ther. 10:146. doi: 10.1186/s13287-019-1223-Z

Becker, L., Schnee, M. E., Niwa, M., Sun, W., Maxeiner, S., Talaei, S., et al. (2018). The presynaptic ribbon maintains vesicle populations at the hair cell afferent fiber synapse. eLife 12:e30241. doi: 10.7554/eLife.30241

Bharadwaj, H. M., Verhulst, S., Shaheen, L., Liberman, M. C., and ShinnCunningham, B. G. (2014). Cochlear neuropathy and the coding of suprathreshold sound. Front. Syst. Neurosci. 8:26. doi: 10.3389/fnsys.2014.00026

Camarero, G., Avendano, C., Fernandez-Moreno, C., Villar, A., Contreras, J., de Pablo, F., et al. (2001). Delayed inner ear maturation and neuronal loss in postnatal IGF-1-deficient mice. J. Neurosci. 21, 7630-7641. doi: 10.1523/JNEUROSCI.21-19-07630.2001

Camarero, G., Villar, M. A., Contreras, J., Fernández-Moreno, C., Pichel, J. G., Avendaño, C., et al. (2002). Cochlear abnormalities in insulin-like growth factor-1 mouse mutants. Hear. Res. 170, 2-11. doi: 10.1016/S03785955(02)00447-1

Coate, T. M., Scott, M. K., and Gurjar, M. (2019). Current concepts in cochlear ribbon synapse formation. Synapse 73:e22087. doi: 10.1002/syn.22087

Fogarty, M. J., Hammond, L. A., Kanjhan, R., Bellingham, M. C., and Noakes, P. G. (2013). A method for the three-dimensional reconstruction of Neurobiotin ${ }^{\mathrm{m}}$ filled neurons and the location of their synaptic inputs. Front. Neural. Circuits 7:153. doi: $10.3389 /$ fncir.2013.00153

Fujiwara, T., Hato, N., Nakagawa, T., Tabata, Y., Yoshida, T., Komobuchi, H., et al. (2008). Insulin-like growth factor 1 treatment via hydrogels rescues cochlear hair cells from ischemic injury. Neuroreport 19, 1585-1588. doi: 10.1097/WNR. 0b013e328311ca4b

Hayashi, Y., Yamamoto, N., Nakagawa, T., and Ito, J. (2013). Insulin-like growth factor 1 inhibits hair cell apoptosis and promotes the cell cycle of supporting cells by activating different downstream cascades after pharmacological hair cell injury in neonatal mice. Mol. Cell. Neurosci. 56, 29-38. doi: 10.1016/j.mcn. 2013.03.003

Hayashi, Y., Yamamoto, N., Nakagawa, T., Omori, K., and Ito, J. (2017). Activation of IGF1 signaling in the cochlea induces the transcription of its mediators during the protection of cochlear hair cells against aminoglycoside. Otol. Neurotol. 38, 278-282. doi: 10.1097/mao.0000000000001276

Hu, N., Rutherford, M. A., and Green, S. H. (2020). Protection of cochlear synapses from noise-induced excitotoxic trauma by blockade of $\mathrm{Ca}^{2+}$-permeable AMPA receptors. Proc. Natl. Acad. Sci. U S A 117, 3828-3838. doi: 10.1073/pnas. 1914247117

Hudspeth, A. J. (1997). How hearing happens. Neuron 19, 947-950. doi: 10.1016/s0896-6273(00)80385-2

Iwai, K., Nakagawa, T., Endo, T., Matsuoka, Y., Kita, T., Kim, T.-S., et al. (2006). Cochlear protection by local insulin-like growth factor-1 application using biodegradable hydrogel. Laryngoscope 116, 529-533. doi: 10.1097/01.mlg. 0000200791.77819.eb

Jenkins, P. J., and Bustin, S. A. (2004). Evidence for a link between IGF-I and cancer. Eur. J. Endocrinol. 151, S17-S22. doi: 10.1530/eje.0.151s017

Kujawa, S. G., and Liberman, M. C. (2009). Adding insult to injury: cochlear nerve degeneration after "temporary" noise-induced hearing loss. J. Neurosci. 29, 14077-14085. doi: 10.1523/jneurosci.284509.2009

Landi, S., Ciucci, F., Maffei, L., Berardi, N., and Cenni, M. C. (2009). Setting the pace for retinal development: environmental enrichment acts through exposed to $50 \mu \mathrm{g} / \mathrm{ml}$ JB1 for $24 \mathrm{~h}$, after which they were incubated with culture media supplemented with rhlGF1 at a concentration of $5 \mu \mathrm{g} / \mathrm{ml}$ or without rhlGF1 for 24 h. (A-D) Maximal-intensity projection images with z-stack of the immunostaining images of specimens cultured with rhlGF1 at concentrations of 0 and $5 \mu \mathrm{g} / \mathrm{ml}$. (E,F) No significant loss of inner hair cell $(\mathrm{IHC})$ or spiral ganglion neuron (SGN) was found with student's $t$-test $p=0.578$ for $\mathrm{IHC}$ and $p=0.133 \mathrm{for}$ SGN). Scale bars: $10 \mu \mathrm{m}$. Data are expressed as mean (digits at the top of each bar) \pm SD. The digits at the bottom of each bar represent the sample number.

insulin-like growth factor 1 and brain-derived neurotrophic factor. J. Neurosci 29, 10809-10819. doi: 10.1523/JNEUROSCI.1857-09.2009

Lee, K. Y., Nakagawa, T., Okano, T., Hori, R., Ono, K., Tabata, Y., et al. (2007). Novel therapy for hearing loss: delivery of insulin-like growth factor 1 to the cochlea using gelatin hydrogel. Otol. Neurotol. 28, 976-981. doi: 10.1097/MAO. 0b013e31811f40db

Li, S., Hang, L., and Ma, Y. (2016). FGF22 protects hearing function from gentamycin ototoxicity by maintaining ribbon synapse number. Hear. Res. 332, 39-45. doi: 10.1016/j.heares.2015.11.011

Liberman, L. D., and Liberman, M. C. (2016). Postnatal maturation of auditorynerve heterogeneity, as seen in spatial gradients of synapse morphology in the inner hair cell area. Hear. Res. 339, 12-22. doi: 10.1016/j.heares.2016. 06.002

Liu, K., Jiang, X., Shi, C., Shi, L., Yang, B., Shi, L., et al. (2013). Cochlear inner hair cell ribbon synapse is the primary target of ototoxic aminoglycoside stimuli. Mol. Neurobiol. 48, 647-654. doi: 10.1007/s12035-0138454-2

Meltser, I., Cederroth, C. R., Basinou, V., Savelyev, S., Lundkvist, G. S., and Canlon, B. (2014). TrkB-mediated protection against circadian sensitivity to noise trauma in the murine cochlea. Curr. Biol. 24, 658-663. doi: 10.1016/j.cub. 2014.01.047

Meyer, A. C., Frank, T., Khimich, D., Hoch, G., Riedel, D., Chapochnikov, N. M., et al. (2009). Tuning of synapse number, structure and function in the cochlea. Nat. Neurosci. 12, 444-453. doi: 10.1038/nn.2293

Michanski, S., Smaluch, K., Steyer, A. M., Chakrabarti, R., Setz, C., Oestreicher, D., et al. (2019). Mapping developmental maturation of inner hair cell ribbon synapses in the apical mouse cochlea. Proc. Natl. Acad. Sci. U S A 116, 6415-6424. doi: 10.1073/pnas.1812029116

Nakagawa, T., Kumakawa, K., Usami, S.-I., Hato, N., Tabuchi, K., Takahashi, M., et al. (2014). A randomized controlled clinical trial of topical insulin-like growth factor-1 therapy for sudden deafness refractory to systemic corticosteroid treatment. BMC Med. 12:219. doi: 10.1186/s12916-0140219-x

Nakagawa, T., Sakamoto, T., Hiraumi, H., Kikkawa, Y. S., Yamamoto, N., Hamaguchi, K., et al. (2010). Topical insulin-like growth factor 1 treatment using gelatin hydrogels for glucocorticoid-resistant sudden sensorineural hearing loss: a prospective clinical trial. BMC Med. 8:76. doi: 10.1186/17417015-8-76

Nakagawa, T., Yamamoto, M., Kumakawa, K., Usami, S., Hato, N., Tabuchi, K., et al. (2016). Prognostic impact of salvage treatment on hearing recovery in patients with sudden sensorineural hearing loss refractory to systemic corticosteroids: a retrospective observational study. Auris Nasus Larynx 43, 489-494. doi: 10.1016/j.anl.2015.12.004

Nelson, B. S., Springer, R. C., and Daniel, J. M. (2014). Antagonism of brain insulin-like growth factor-1 receptors blocks estradiol effects on memory and levels of hippocampal synaptic proteins in ovariectomized rats. Psychopharmacology 231, 899-907. doi: 10.1007/s00213-0133310-7

Okano, T., Xuan, S., and Kelley, M. W. (2011). Insulin-like growth factor signaling regulates the timing of sensory cell differentiation in the mouse cochlea. J. Neurosci. 31, 18104-18118. doi: 10.1523/JNEUROSCI.361911.2011

Pietrzkowski, Z., Wernicke, D., Porcu, P., Jameson, B. A., and Baserga, R. (1992). Inhibition of cellular proliferation by peptide analogues of insulin-like growth factor 1. Cancer Res. 52, 6447-6451.

Riquelme, R., Cediel, R., Contreras, J., Rodriguez-de la Rosa, L., Murillo-Cuesta, S., Hernandez-Sanchez, C., et al. (2010). A comparative study of age-related 
hearing loss in wild type and insulin-like growth factor I deficient mice. Front. Neuroanat. 4:27. doi: 10.3389/fnana.2010.00027

Safieddine, S., El-Amraoui, A., and Petit, C. (2012). The auditory hair cell ribbon synapse: from assembly to function. Annu. Rev. Neurosci. 35, 509-528. doi: 10.1146/annurev-neuro-061010-113705

Sanchez-Calderon, H., Rodriguez-de la Rosa, L., Milo, M., Pichel, J. G., Holley, M., and Varela-Nieto, I. (2010). RNA microarray analysis in prenatal mouse cochlea reveals novel IGF-I target genes: implication of MEF2 and FOXM1 transcription factors. PLoS One 5:e8699. doi: 10.1371/journal.pone. 0008699

Suzuki, J., Corfas, G., and Liberman, M. C. (2016). Round-window delivery of neurotrophin 3 regenerates cochlear synapses after acoustic overexposure. Sci. Rep. 6:24907. doi: 10.1038/srep24907

Vandenberg, L. N., Colborn, T., Hayes, T. B., Heindel, J. J., Jacobs, D. R. Jr., Lee, D.-H., et al. (2012). Hormones and endocrine-disrupting chemicals: low-dose effects and nonmonotonic dose responses. Endocr. Rev. 33, 378-455. doi: 10.1210/er.2011-1050

Varela-Nieto, I., Hartl, M., Gorospe, I., and Leon, Y. (2007). Anti-apoptotic actions of insulin-like growth factors: lessons from development and implications in neoplastic cell transformation. Curr. Pharm. Des. 13, 687-703. doi: $10.2174 / 138161207780249164$

Viana, L. M., O’Malley, J. T., Burgess, B. J., Jones, D. D., Oliveira, C. A., Santos, F., et al. (2015). Cochlear neuropathy in human presbycusis: confocal analysis of hidden hearing loss in post-mortem tissue. Hear. Res. 327, 78-88. doi: 10.1016/j.heares.2015.04.014

Wagner, E. L., and Shin, J.-B. (2019). Mechanisms of hair cell damage and repair. Trends Neurosci. 42, 414-424. doi: 10.1016/j.tins.2019.03.006

Wan, G., Gómez-Casati, M. E., Gigliello, A. R., Liberman, M. C., and Corfas, G. (2014). Neurotrophin-3 regulates ribbon synapse density in the cochlea and induces synapse regeneration after acoustic trauma. eLife 3:e03564. doi: $10.7554 /$ eLife. 03564

Wang, Q., and Green, S. H. (2011). Functional role of neurotrophin-3 in synapse regeneration by spiral ganglion neurons on inner hair cells after excitotoxic trauma in vitro. J. Neurosci. 31, 7938-7949. doi: 10.1523/jneurosci.1434-10. 2011

World Health Organization (2018). Deafness and Hearing Loss. World Health Organization Fact Sheets. Available online at: https://www.who.int/en/newsroom/fact-sheets/detail/deafness-and-hearing-loss

Yamahara, K., Nakagawa, T., Ito, J., Kinoshita, K., Omori, K., and Yamamoto, N. (2017). Netrin 1 mediates protective effects exerted by insulin-like growth factor 1 on cochlear hair cells. Neuropharmacology 119, 26-39. doi: 10.1016/j. neuropharm.2017.03.032

Yamahara, K., Asaka, N., Kita, T., Kishimoto, I., Matsunaga, M., Yamamoto, N., et al. (2019). Insulin-like growth factor 1 promotes cochlear synapse regeneration after excitotoxic trauma in vitro. Hear. Res. 374, 5-12. doi: 10.1016/j.heares.2019.01.008

Yamahara, K., Nishimura, K., Ogita, H., Ito, J., Nakagawa, T., Furuta, I., et al. (2018). Hearing preservation at low frequencies by insulin-like growth factor 1 in a guinea pig model of cochlear implantation. Hear. Res. 368, 92-108. doi: 10.1016/j.heares.2018.07.004

Zuccotti, A., Kuhn, S., Johnson, S. L., Franz, C., Singer, W., Hecker, D., et al. (2012). Lack of brain-derived neurotrophic factor hampers inner hair cell synapse physiology, but protects against noise-induced hearing loss. J. Neurosci. 32, 8545-8553. doi: 10.1523/JNEUROSCI.1247-12.2012

Conflict of Interest: The authors declare that the research was conducted in the absence of any commercial or financial relationships that could be construed as a potential conflict of interest.

Copyright $(2020$ Gao, Kita, Katsuno, Yamamoto, Omori and Nakagawa. This is an open-access article distributed under the terms of the Creative Commons Attribution License (CC BY). The use, distribution or reproduction in other forums is permitted, provided the original author(s) and the copyright owner(s) are credited and that the original publication in this journal is cited, in accordance with accepted academic practice. No use, distribution or reproduction is permitted which does not comply with these terms. 